\section{Fecal microbiota transplantation: the state of the art}

\section{Stefano Di Bella, ${ }^{1}$ Cecilia Drapeau, ${ }^{2}$ Esther García-Almodóvar, ${ }^{3}$ Nicola Petrosillo'}

'National Institute for Infectious Diseases L. Spallanzani, Rome, Italy; ${ }^{2}$ Department of Medical Microbiology, King's College Hospital, London, UK; ${ }^{3}$ Department of Internal Medicine, Son Espases University Hospital, Palma of Majorca, Spain

\begin{abstract}
Clostridium difficile infection (CDI) is an emerging problem in terms of incidence, morbidity and mortality. Currently available treatment options are not always effective, especially in cases of recurrent/refractory or complicated CDI. The gut microbiota transplantation is a technique that has been sporadically practiced since the ' $50 \mathrm{~s}$, but its clinical efficacy has only recently been supported by scientific evidence. In the present article, we report the pathophysiological basis and the clinical indications of this technique that, in light of its low cost, and proven efficacy and safety, is likely to become part of the management guidelines of difficult cases of CDI in the near future.
\end{abstract}

\section{Introduction}

The epidemiology of Clostridium difficile infection (CDI) is pointing towards a significant increase in morbidity and mortality rates, especially in developed countries. Moreover, the spread of ribotype NAP1/BI/027 and other hypervirulent strains has contributed to make the management of this type of infection even more difficult.

An emerging issue, which represents a real challenge for the clinicians, is the management of recurrent cases of CDI. About $20 \%$ of patients with CDI experience a recurrent episode after initial antibiotic treatment. Patients with a recurrent episode have 40\% chance of experiencing another one, while in those who have $\geq 2$ recurrent episodes the likelihood of a further one reaches $60 \%{ }^{2,3}$

To date, there is still no standardized treatment for patients with recurrent/relapsing episodes of CDI, and usually these patients are committed to several courses of antiClostridium difficile antibiotics. In these cases, the signs and symptoms of CDI usually reappear after discontinuation of antibiotic treatment. Actually, the current international guidelines declare the existence of a gap in the management of these clinical situations. ${ }^{4}$

Unconventional therapies have been proposed as alternative or adjunctive treatment to the classic metronidazole and vancomycin, such as the use of intravenous immunoglobulin, and others like probiotics, and chelating agents. However, the results in terms of clinical cure have been far from satisfactory. In light of these therapeutic limitations the technique of fecal bacteriotherapy was rediscovered, with the rationale of a real organ transplant. The first reported fecal transplant in humans in the literature dates back to $1958 .^{5}$ The use of fecal bacteriotherapy was in fact reported in the literature for about 50 years, but until 2013 no randomized trial was ever been published.

Over the last few years, the term intestinal flora (now considered obsolete) has been gradually replaced with that of gut microbiota, thus indicating the growing awareness of the existence of an actual organ responsible of multiple physiological functions (i.e. energy metabolism and immune system), similarly to what has happened with the adipose tissue in metabolic diseases.

\section{Pathophysiological substrate of Clostridium difficile infection: the disruption of the intestinal microbiota}

Chang et al. analyzed the fecal microbiota of 7 patients with CDI using the 16S rDNA sequencing, and found a progressive reduction in the diversity of the bacterial species in patients with initial CDI, in patients with recurrent $\mathrm{CDI}$ and in healthy controls. ${ }^{6}$ In particular, among CDI patients, there is a marked reduction in Bacteroides and an increase in the Proteobacteria.

A subsequent study of Khoruts et al. compared the composition of the intestinal microbiota in CDI patients before and after the fecal bacteriotherapy. ${ }^{7}$ Patients with CDI showed a smaller amount of Firmicutes and Bacteroides; however, 14 days after the procedure of the fecal transplant, a significant change in the composition of the microbiota occurred, with a bacterial composition once again dominated by Bacteroides, resembling that of healthy individuals.

In a recent randomized clinical trial on duodenal infusion of feces for the treatment of recurrent $\mathrm{CDI}$, an analysis of fecal microbiota using $16 \mathrm{~S}$ rRNA before and after the infusion of feces is also described. ${ }^{8}$ In agreement with
Correspondence: Stefano Di Bella, National Institute for Infectious Diseases L. Spallanzani, via Portuense 292, 00149 Rome, Italy.

Tel. +39.06.551.70294 - Fax: +39.06 .551 .70486 .

E-mail: stefano932@gmail.com

Key words: Clostridium difficile infection, microbiota transplantation.

Contributions: the authors contributed equally.

Conflict of interests: the authors declare no potential conflict of interests.

Received for publication: 4 June 2013.

Accepted for publication: 4 October 2013

This work is licensed under a Creative Commons Attribution NonCommercial 3.0 License (CC BYNC 3.0).

(C) Copyright S. Di Bella et al., 2013

Licensee PAGEPress, Italy

Infectious Disease Reports 2013; 5:e13

doi:10.4081/idr.2013.e13

the above studies, these authors also observe that, after the infusion of feces from healthy donors, the fecal bacterial diversity grows back, with an increase in Bacteroides and Clostridium clusters IV and XIV and a decrease in the Proteobacteria.

\section{Clinical use of the intestinal microbiota transplantation: scientific evidence}

The practical procedure of transplantation of intestinal microbiota consists of an instillation of a liquid suspension of stool from a healthy donor to a patient with CDI. The liquid is placed in the gastrointestinal tract by nasogastric/nasojejunal tube, gastroscope, colonscope or a retention enema. The literature from 1958 to 2008 describes case reports and case series of 100 episodes of recurrent CDI treated with fecal bacteriotherapy with a success rate of $89 \%{ }^{9}$

In 2011, a systematic review of the literature on 317 patients with recurrent CDI treated with transplantation of intestinal microbiota was published. ${ }^{10}$ Ninety-two percent $(92 \%)$ of patients had resolution of symptoms $(89 \%$ after a single treatment and $5 \%$ after retreatment), and $4 \%$ showed recurrence of symptoms after transplantation. There were 13 (4\%) deaths during follow-up, 3 of which, all from the same study, attributed to CDI (1\%). None of the studies reported in this systematic review was a randomized clinical trial.

In 2013 the first randomized trial on the infusion of feces for the treatment of recurrent 
ICD was published. ${ }^{8}$ The trial compared 3 groups of patients: patients treated with oral vancomycin followed by intestinal lavage followed by infusion of feces through the gastroscope; oral vancomycin alone; and oral vancomycin followed by intestinal lavage. The primary endpoint was cure without relapse within 10 weeks after the initiation of therapy. Cure was defined as absence of diarrhea or persistent diarrhea that could be explained by other causes with 3 consecutive negative toxin tests for $C$. difficile. The trial was stopped after an interim analysis in which 15 of 16 (93\%) patients in the group treated with infusion of feces had resolution of diarrhea (13 after the first infusion and 2 after the second infusion) compared to 4 out of $13(30.7 \%)$ of those who received vancomycin alone, and only 3 out of $13(23 \%)$ of those who received vancomycin and intestinal lavage $(\mathrm{P}<0.001)$.

In order to better implement this technique, the characteristics of the donor and recipient should be carefully assessed.

\section{Donor}

\section{Inclusion criteria}

In light of the recent evidence about the involvement of the intestinal microbiota in systemic non-infectious diseases, the candidate donor has to be preferably a healthy volunteer. Until 2011 , a partner or a family member was the most frequent donor; however, the reasons supporting this option were not evidence based. Recently, a pool of periodically screened donors has been built for safety, cost and speed of disposal of fecal material ready to be transplanted. ${ }^{8,11}$ Some authors prefer the younger donors, and the males to the females.

\section{Exclusion criteria}

Exclusion criteria in fecal donors are similar to those universally used in blood products donors plus a few other specific criteria for CDI. A dedicated questionnaire should contain the following items: known HIV or HCV or HBV infection, known exposure to HIV or viral hepatitis within the previous 12 months, high-risk sexual behaviors, use of illicit drugs, tattoo or body piercing within 6 months, incarceration or history of incarceration, known current communicable disease, risk factors for variant Creutzfeldt-Jacob disease, travel (within the last 6 months) to areas of the world where enteric pathogens are endemic or the risk of traveller's diarrhea is high, history of inflammatory bowel disease, history of inflammatory bowel syndrome, idiopathic chronic constipation, or chronic diarrhea, history of gastrointestinal malignancy or known polyposis, antibiotics within the preceding 3 months, major immunosoppressive medications, sistemic antineoplastic agents, recent ingestion of a potential allergen.

Recently, in light of the evidence supporting a role of the intestinal microbiota in systemic diseases, some authors report also the following relative contraindications: history of major gastrointestinal surgery, metabolic syndrome, sistemi autoimmunities, allergic diseases, eosinophilic disorders of the gastrointestinal tract, chronic pain syndromes (e.g. chronic fatigue syndrome, fibromyalgia).

\section{Donor screening}

The data in literature show that the number of complications related to the procedure of fecal infusion is very low. The most widely feared potential risk remains that of the transmission of infectious agents from the donor. For this reason a careful screening of the donor is required. There is no standard screening for donor, since this depends on the resources of the hospital and on the degree of risk that is considered acceptable (in a totally comparable way to what happens for transfusion of blood products between different nations). However, for practical reasons we report in Table 1 the various infectious agents screened for in the donor testing used in the above-mentioned recent Dutch randomized trial. ${ }^{8}$

\section{Recipient}

\section{Inclusion criteria}

The ideal recipient candidates are patients older than 18 years with recurrent CDI and failure to at least one cycle of appropriate antibiotic therapy. The number of recurrent episodes necessary to include the patient is still to be determined; in the Dutch trial one was enough, while other studies only consider patients with at least 2 episodes of severe CDI or 3 of episodes of mild or moderate CDI. ${ }^{8,12}$ Moreover, application of this technique is still to be evaluated in cases of non-responders to standard antibiotic therapy and in those with fulminant Clostridium difficile colitis.

\section{Exclusion criteria}

Exclusion criteria are actually subject to variability from center to center, and so far there are no standardized exclusion criteria. However, it is important to assess, among the potential recipients for transplantation of feces, those at increased risk of adverse events. In literature, immunocompromized patients are considered to be at greater risk.
However, this assumption is dictated more by common sense and evidence deriving from other types of infections than from scientific evidence specific to $C$. difficile. The most commonly used exclusion criteria in literature are: i) patients on major immunosoppressive agents including high-dose corticosteroids, calcineurin inhibitors, mammalian target of rapamycin (mTOR) inhibitors, lymphocitedepleting biological agents, anti-tumor necrosis factor agents, and others, chemotherapeutic antineoplastic treatments; ii) patients with decompensated liver cirrhosis, advanced HIV/acquired immune deficiency syndrome, recent bone marrow transplant, or other causes of severe immunodeficiency.

Among the exclusion criteria, some authors consider also pregnancy, ICU admission or treatment with vasopressors; these criteria will be likely reviewed in the future, especially if the fecal transplant is proposed as an alternative to colectomy in fulminant cases. Moreover, patients with low life expectancy are usually excluded..$^{8,11}$

\section{Preparation of stool}

The fecal material is usually collected from the donor and filtered to remove the particulate matter (e.g. undigested fibers, etc.). Many authors dilute the filtered material with saline solution until they obtain a volume of $25-50 \mathrm{~mL}$ and $250-500 \mathrm{~mL}$ for administration via the upper gastrointestinal tract and the lower one, respectively. ${ }^{12}$

Until recently, it was preferred to collect and infuse the feces within 24 hours (preferably 6 hours) and the material was not frozen. Recently, Hamilton et al. have shown that the use of a bank of fecal material properly processed and frozen represents a viable option to be used as needed using samples already analyzed and ready for use. ${ }^{11}$

Table 1. Donor screening.

\begin{tabular}{ll} 
Blood & Stool \\
HIV & Parasites \\
HTLV 1-2 & C. difficile \\
HAV, HBV, HCV & Enteropathogenic bacteria \\
EBV, CMV & \\
Treponema pallidum & \\
Strongyloides stercoralis & Entamoeba histolytica \\
\hline
\end{tabular}

Adapted from van Nood et al. 2013. ${ }^{8}$ 


\section{Pretreatment of recipient}

The recipient is usually given a bowel preparation like the one used for colonoscopy with the rationale to potentially remove pre-existing flora (pathological) and spores of $C$. difficile prior to instillation of feces. If the infusion is via nasogastric (or naso-jejunal) tube, some authors administer a proton pump inhibitor the night before and the morning of the procedure. ${ }^{12,13}$ Some authors use loperamide if the stools are administered by a colonoscope or retention enema. ${ }^{14}$

Many authors keep the patient on oral vancomycin until the time of transplantation. ${ }^{8,13}$ The rationale for pretreating patients with vancomycin is to reduce the amount of vegetative forms of $C$. difficile (vancomycin has no action on the spores). Although this practice is widely used and rationally valid, there is no evidence of different efficacy comparing pretreated patients and treatment-naïve patients. The same is true with regards to the bowel preparation before the transplant procedure.

\section{Instillation route}

The best instillation route is still a controversial topic. Until 1989 enema was the most commonly used route, and then gradually other instruments were utilized such as nasogastric tube (1991), colonoscopy (2000) and the selfadministered enema (2010). ${ }^{15}$ To date, more than 400 cases of patients with fecal transplant have been reported, approximately $75 \%$ of which by colonoscopy or barium enema, and $25 \%$ by nasogastric or nasojejunal tube or gastroscope. $^{15}$

Although the risks associated with the microbiota transplant procedure are very limited, clinicians should be aware that the instillation of feces through nasogastric tube could potentially cause aspiration of fecal material, and therefore it is reasonable to consider the need of an X-ray to ensure that the tube is in place. On the other hand, the contrast barium enema and the colonoscopy have the risk of perforation (especially in patients with underlying diseases such as inflammatory bowel disease and/or diverticulosis).

One study compared the effectiveness of fecal transplantation on the basis of the route of administration (nasogastric vs. colonoscopic) through a review of studies published in literature. ${ }^{16}$ The authors found no differences in efficacy between the two techniques.

\section{Conclusions}

Fecal transplantation represents a therapy with a high potential, and its rationale, though seemingly simple, opens up to a wide range of investigation fields. The microbiota transplantation has shown to have a role in many diseases, not only infectious: inflammatory bowel diseases, irritable bowel syndrome, obesity and metabolic syndromes, anorexia nervosa, autoimmunity, and multiple sclerosis. ${ }^{17}$ Recently, the microbiota has also been shown to be involved in the production of metabolites (proatherosclerotic metabolite, trimethylamine- $\mathrm{N}$-oxide) associated with an increased risk of major cardiovascular events. ${ }^{18}$

In conclusion, as regards the management of recurrent forms of CDI, transplantation of intestinal microbiota represents a relatively simple procedure, of short duration and with a high clinical cure rate. The risks associated with this procedure seem to be limited, especially if the criteria for inclusion and exclusion are contemplated and the donor screening tests are performed. The cost of transplantation of intestinal microbiota is bound to be lower if compared to the cost of repeated courses of antibiotic therapy, hospitalization, as well as the loss of work productivity caused by the persistence of diarrhea. ${ }^{19}$

This new therapeutic option will not only be used effectively in the near future, it will also open new interesting investigation fields.

\section{References}

1. Freeman J, Bauer MP, Baines SD, et al. The changing epidemiology of Clostridium difficile infections. Clin Microbiol Rev 2010;23:529-49.

2. McFarland LV, Elmer GW, Surawicz CM. Breaking the cycle: treatment strategies for 163 cases of recurrent Clostridium difficile disease. Am J Gastroenterol 2002;97: 1769-75.

3. Kelly CP, LaMont JT. Clostridium difficile more difficult than ever. $\mathrm{N}$ Engl $\mathrm{J}$ Med 2008;359:1932-40.

4. Cohen SH, Gerding DN, Johnson S, et al. Clinical practice guidelines for Clostridium difficile infection in adults: 2010 update by the society for healthcare epidemiology of America (SHEA) and the infectious diseases society of America (IDSA). Infect Control Hosp Epidemiol 2010;31:431-55.

5. Eiseman B, Silen W, Bascom GS, Kauvar AJ. Fecal enema as an adjunct in the treatment of pseudomembranous enterocolitis. Surgery 1958;44:854-9.

6. Chang JY, Antonopoulos DA, Kalra A, et al.
Decreased diversity of the fecal microbiome in recurrent Clostridium difficile-associated diarrhea. J Infect Dis 2008;197:4358.

7. Khoruts A, Dicksved J, Jansson JK, Sadowsky MJ. Changes in the composition of the human fecal microbiome after bacteriotherapy for recurrent Clostridium difficile-associated diarrhea. J Clin Gastroenterol 2010;44:354-60.

8. van Nood E, Vrieze A, Nieuwdorp M, et al. Duodenal infusion of donor feces for recurrent Clostridium difficile. N Engl J Med 2013;368:407-15.

9. Bakken JS. Fecal bacteriotherapy for recurrent Clostridium difficile infection. Anaerobe 2009;15:285-9.

10. Gough E, Shaikh H, Manges AR. Systematic review of intestinal microbiota transplantation (fecal bacteriotherapy) for recurrent Clostridium difficile infection. Clin Infect Dis 2011;53:994-1002.

11. Hamilton MJ, Weingarden AR, Sadowsky MJ, Khoruts A. Standardized frozen preparation for transplantation of fecal microbiota for recurrent Clostridium difficile infection. Am J Gastroenterol 2012;107: 761-7.

12. Bakken JS, Borody T, Brandt LJ, et al. Treating Clostridium difficile infection with fecal microbiota transplantation. Clin Gastroenterol Hepatol 2011;9:1044-9.

13. Aas J, Gessert CE, Bakken JS. Recurrent Clostridium difficile colitis: case series involving 18 patients treated with donor stool administered via a nasogastric tube. Clin Infect Dis 2003;36:580-5.

14. Persky SE, Brandt LJ. Treatment of recurrent Clostridium difficile-associated diarrhea by administration of donated stool directly through a colonoscope. Am J Gastroenterol 2000;95:3283-5.

15. Aroniadis OC, Brandt LJ. Fecal microbiota transplantation: past, present and future. Curr Opin Gastroenterol 2013;29:79-84.

16. Postigo R, Kim JH. Colonoscopic versus nasogastric fecal transplantation for the treatment of Clostridium difficile infection: a review and pooled analysis. Infection 2012;40:643-8.

17. Borody TJ, Khoruts A. Fecal microbiota transplantation and emerging applications. Nat Rev Gastroenterol Hepatol 2012;9:88-96.

18. Tang WH, Wang Z, Levison BS, et al. Intestinal microbial metabolism of phosphatidylcholine and cardiovascular risk. N Engl J Med 2013;368:1575-84.

19. Kyne L, Hamel MB, Polavaram R, Kelly CP. Health care costs and mortality associated with nosocomial diarrhea due to Clostridium difficile. Clin Infect Dis 2002; 34:346-53. 